\title{
Italique Itaque
}

Poésie italienne de la Renaissance

II | 1999

Varia

De l'équivoque érotique dans la poésie italienne de la Renaissance, et de l'érotisme discret de l'Arioste en particulier

Paul Larivaille

\section{(2) OpenEdition}

Journals

Édition électronique

URL : https://journals.openedition.org/italique/196

DOI : 10.4000/italique. 196

ISSN : 1663-4438

Éditeur

Librairie Droz

Édition imprimée

Date de publication : 1 décembre 1999

Pagination : 33-53

ISBN : 2-600-00389-4

ISSN : 1423-3983

Référence électronique

Paul Larivaille, « De l'équivoque érotique dans la poésie italienne de la Renaissance, et de l'érotisme discret de l'Arioste en particulier », Italique [En ligne], II | 1999, mis en ligne le 07 octobre 2009, consulté le 26 novembre 2022. URL : http://journals.openedition.org/italique/196 ; DOI : https:// doi.org/10.4000/italique.196 
P A U L L A R IV A I L L E

D E L'É Q U IV O Q U E ÉR O T I Q E D A N S A POÉSIE I T A L I N N E DE LA RENAISSA NCE,

E T D E L'ÉR OT IS M E D I S C RET DE L'ARIOSTE EN PARTICULIER ${ }^{1}$ 


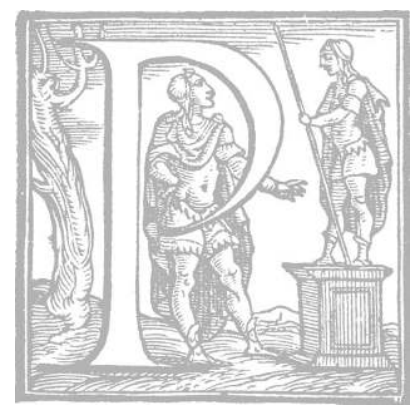

Our ne pas remonter trop loin et m'en tenir au domaine de la poésie italienne, je me limiterai, en guise d'entrée en matière, à rappeler la conclusion de la cinquième journée du Décaméron, où un des conteurs, Dioneo, prié par la nouvelle reine de chanter une chanson, avant de se résoudre à "laisse[r] là les bêtises» et en chanter "une belle», énumère les premiers vers de pas moins de neuf ballades licencieuses, dont les éclats de rire des sept jeunes femmes présentes indiquent à l'évidence que la suite ne leur est pas inconnue.

Ce type de composition, qui n'était certes pas nouveau au temps de Boccace, atteindra au comble du raffinement dans les vers des poètes florentins $d u X V^{e}$ siècle, parmi lesquels, dans une thèse monumentale devenue en moins de vingt années un ouvrage de référence incontournable, Jean Toscan, qui a été le premier à déchiffrer correctement beaucoup de textes jusque-là mal ou pas compris, a distingué deux courants: l'un, plus agressif, illustré par les sonnets du barbier Burchiello, mort en exil à Rome en I449; l'autre, où prédomine "l'amour du jeu », qui trouve son épanouissement dans l'entourage de Laurent le Magnifique, et en tout premier lieu dans certaines canzoni a ballo (littéralement "chansons à danser", c'est-à-dire ballades) ou chants de carnaval de Laurent lui-même. ${ }^{2}$ Le jeu, une gageure fondée sur l'exploitation systématique de l'équivoque, consiste à superposer aussi parfaitement que possible - comme dans une allégorie réussie - deux niveaux de lecture du texte: l'un normal, voire innocent ou anodin, en tout cas ordinaire et accessible à tous; l'autre obscène, accessible à des initiés, seuls en mesure de comprendre le sens caché des mots et des formules employés; un sens - si tant est qu'il ne soit pas resté pendant des siècles insoupçonné des commentateurs - demeuré parfois si hermétique à l'exégèse, que seules les patientes recherches de Toscan ont permis de le mettre au jour. De Florence, où Machiavel s'y essaiera lui aussi avec quelque bonheur dans un ou deux de ses propres chants de carnaval, la poésie «équivoquée » s'exportera à partir de ISIz à Rome, à la faveur de l'élection au pontificat de Léon X-fils cadet de Laurent le Magnifique notoirement friand de facéties de toutes sortes, comme, pour ne prendre que ce seul exemple qui nous rapproche de notre propos, les doubles sens obscènes sur lesquels s'ouvre le prologue des Suppositi - une comédie de l'Arioste jouée en sa présence pendant le carnaval de I5I9 - qui le feront "ri[re] fort gaillardement avec les autres spectateurs ». ${ }^{3}$ Et c'est à Rome que Francesco Berni, un jeune poète arrivé de Toscane en III7 au service du cardinal Bibbiena lui-même auteur quelques années plus tôt d'une comédie facétieuse non exempte de quelques jeux salaces -, achèvera de perfectionner le genre, faisant 
durablement école dans la péninsule, où, jusqu'au XVIII siècle, nombre de poètes burlesques se targueront de composer «à la bernesque ${ }^{4}{ }^{4}$

La clé du "langage de l'équivoque - en vers ou en prose - réside, comme le rappelle justement Toscan, dans l'exploitation de l'euphémisme: "une figure - pour reprendre la définition de Du Marsais, où il suffit de remplacer le mot "idée » par celui d" "image » pour l'approprier parfaitement au cas de la littérature érotique - par laquelle on déguise des idées désagréables, odieuses, ou tristes, sous des noms qui ne sont point les noms propres de ces idées; ils leur servent comme de voiles, et ils en expriment en apparence de plus agréables, de moins choquantes, ou de plus honnêtes selon le besoin ». ' En l'occurrence, l'euphémisme - étymologiquement le "bien parler» - devant camoufler sous des apparences de bienséance un nombre tout compte fait limité d'évocations ou de descriptions d'organes et d'actes sexuels, la pratique assidue de l'équivoque érotique devient vite un pur jeu de langage, dont les adeptes n'ont d'autre solution pour se distinguer, que de faire perpétuellement assaut de virtuosité verbale, explorant de toujours nouveaux termes de substitution, dans une sorte de défi mutuel permanent qui les conduit, de surenchère en surenchère, à explorer toujours plus en détail non seulement le monde quotidien, mais tout un univers géographique et cosmique : mobilisant ainsi une part de plus en plus grande des champs lexicaux courants, au risque - et parfois avec l'intention évidente - de dérouter le profane, d'accroitre l'hermétisme des textes et d'en réserver la jouissance à une élite restreinte de privilégiés.

Si cette poésie de l'équivoque, mise au point pour l'essentiel à Florence, et dans la Florence médicéenne de la fin du $X V^{e}$ siècle en particulier, puis, grâce au talent exceptionnel de Berni, devenue un genre plus ou moins habilement cultivé à Rome et ailleurs par des générations de poètes burlesques, atteste bien la permanence et la profondeur d'un penchant pour l'érotisme répandu jusque dans les couches les plus élevées et cultivées de la société italienne de la Renaissance, il s'en faut pourtant de beaucoup qu'elle suffise à rendre compte de l'ampleur du phénomène et à en épuiser les champs et les modes de manifestation. Même si elle tend toujours plus - ce qui explique sans doute en partie qu'elle ait continué tant bien que mal à fleurir au temps des rigueurs de la Contre-Réforme triomphante - vers un hermétisme ludique où l'obscénité elle-même finit par s'estomper et devenir invisible à un xil non exercé, une connaissance précise des mécanismes qu'elle met en auvre, et que Toscan a bien mis en lumière, est indispensable: en premier lieu, pour corriger les erreurs des critiques, et non des moindres, à qui, ne comprenant pas un sens caché dont ils ne soupçonnaient parfois même pas l'existence, il est arrivé de prendre des vessies pour des lanternes et, soit de mépriser pour leur platitude, soit de prendre ingénument pour argent comptant des "allégations de premier 
ÉROTISME DISCRET DE L'ARIOSTE

niveau » dont rien ne prouve la crédibilité ${ }^{6}$; mais aussi et surtout pour faciliter et parfois éclairer la compréhension d'une foule d'autres textes, faisant appel, la plupart du temps sporadiquement, à des euphémismes traditionnels généralement accessibles, mais dont le lecteur n'est jamais certain de saisir pleinement les éventuelles nuances. Car, sans parler de l'exception que constituent les Sonnets luxurieux de l'Arétin, une bravade délibérée, sans le moindre travestissement verbal, probablement non étrangère aux coups de poignard qui marquent la fin de la carrière romaine de leur auteur en I525, la littérature de la Renaissance italienne est riche en facéties volontiers licencieuses dont les euphémismes n'ont d'autre prétention que d'atténuer la verdeur de l'expression sans dissimuler le sens second des textes. S'agissant en effet d'cuvres destinées non plus, comme la poésie équivoquée, à un cercle restreint d'amateurs et souvent concurrents, mais à un vaste public, les auteurs ne peuvent aller trop au-delà d'un code minimal, connu et compris de tous, qui à l'époque coïncide souvent peu ou prou, d'un bout à l'autre de l'Italie, avec le lexique érotique bérité de Boccace: l'écrivain dont matière et langage sont les plus présents, non seulement dans la prose narrative, mais dans les comédies, où trames, situations et personnages inspirés du Décaméron se conjuguent d'abord à l'béritage de Plaute et de Térence - comme dans la Calandria du cardinal Bibbiena - avant de devenir prépondérants - comme dans la Mandragore machiavélienne -; et jusque dans les poèmes chevaleresques, à tout le moins dans ces sortes d'intermèdes narratifs parfois sans grand rapport avec l'action centrale que l'on a précisément coutume d'appeler des "nouvelles》. Dès lors qu'il ne s'agit plus à proprement parler d'occulter l'obscénité, mais simplement de la recouvrir d'un voile transparent destiné à pimenter descriptions et situations sans outrepasser les limites formelles de la décence, ${ }^{7}$ le problème, pour les auteurs, n'est plus de ne contrevenir en rien à la bienséance, mais de savoir jusqu'où ils peuvent ne pas aller trop loin. On peut se faire une idée de l'ampleur des licences de langage ainsi admissibles - et admises - dans les premières décennies du XVI ${ }^{e}$ siècle à la lecture, par exemple, des comédies que l'A rioste compose pour le public de la cour de Ferrare: de ces jeux de mots à sous-entendus obscènes, métaphores hardies ou calembours équivoques, dont s'émaillent certaines scènes de ses pièces, de la Cassaria, présentée au carnaval de I508, jusqu'à la Lena écrite une vingtaine d'années plus tard. ${ }^{8}$ Mais les documents les plus probants sont sans doute les deux volumes des Ragionamenti de l'Arétin, 9 et en particulier la première des six journées, où, proclamant que "l'bonnêteté est belle dans un lupanar", il s'applique à camoufler systématiquement le langage de l'amour sous des périphrases, comparaisons et surtout métaphores transparentes, suggérant en réalité beaucoup plus que les mots les plus crus qu'il avait employés quelques années plus tôt dans ses Sonnets luxurieux. 
C'est seulement au cours de la décennie suivant la disparition de l'Arioste (I533) et la publication des Ragionamenti (I534-36) que commencera à être battue en brèche avec une vigueur croissante la tolérance qui avait jusque-là permis aux auteurs de déjouer ou tourner, par un langage formellement surveillé, les interdits de la morale chrétienne. Dès avant son achèvement, au début des années I540, le Jugement dernier de Michel-Ange sera l'objet d'attaques dont l'Arétin lui-même, par ressentiment personnel envers l'artiste, n'bésitera pas à se faire l'écho dans une lettre de novembre I545 restée fameuse, opposant cyniquement le «langage irréprébensible et chaste 》 dont il se vante d'avoir affublé la «matière lascive et impudique » de ses Ragionamenti aux " anges » et aux « saints» du maître, "ces derniers sans la moindre terrestre bonnêteté, les autres dépourvus de tout céleste ornement». ${ }^{10}$ Et lorsqu'en I564, au lendemain de la mort de l'artiste, une commission issue du Concile de Trente fera recouvrir les parties honteuses des nus de Michel-Ange, une telle décision ne fera que confirmer avec éclat la mainmise désormais inéluctable de la censure de la Contre-Réforme non seulement sur l'art religieux, mais sur la littérature profane, soumise, depuis la parution du premier Index des livres interdits (I557), aux rigueurs croissantes de l'Inquisition.

Ensuite, non seulement un langage formellement châtié ne suffira plus à préserver un auteur de la censure, mais les contenus eux-mêmes, devront ajouter à l' "honnêteté » un strict respect du dogme et des convenances propres au genre et au sujet traité. Ainsi l'amour, toléré (dans les limites imposées par l'bonnêteté) dans un genre mondain comme la pastorale, ne pourra-t-il que devenir suspect dans un poème hérö̈que mettant en jeu la religion chrétienne comme la Jérusalem délivrée ; et le Tasse devra s'évertuer à légitimer sa présence, avant de se convaincre lui-même plus tard, submergépar les scrupules religieux, de la nécessité d'exclure de sa Jérusalem conquise les épisodes les plus critiqués par les réviseurs de la première version de son poème.

Même si le Tasse, partagé entre la hantise de l'bérésie et une expérience personnelle de la sensualité dont son imagination et son style ne pourront que porter les marques, fera - à son corps défendant parfois - une large place à l'érotisme, il ne pourra s'agir que d'un érotisme contenu, ambigu, trouble, fort éloigné des camouflages transparents, plus ludiques que franchement voluptueux, qui avaient encore cours quelques décennies plus tôt, au temps où l'Arioste composait son Roland furieux. ${ }^{\text {"I }}$ Tant il est vrai que le futur poète de la Jérusalem délivrée se sentira tenu, dès ses juvéniles Discours de l'art poétique, de réprouver les hardiesses incongrues à ses yeux de certains passages du poème de l'Arioste: formulant déjà, sous le couvert de considérations théoriques, des reproches qu'il réitérera en des termes plus mordants près de trente ans plus tard, au livre IV de ses Discours du poème héroïque, où, par delà le style et les vers ponctuellement cités, la réprobation s'attachera 
ÉROTISME DISCRET DE L'ARIOSTE

avec plus d'évidence à la matière de scènes et d'épisodes entiers jugés inconvenants sous la plume d'un "poète béroüque : non seulement par ce qu'ils donnent à voir, mais par ce que simplement ils suggèrent (" la cosa [...] che s'accenna »). ${ }^{\mathrm{I}}$

C'est à partir de la base objective ainsi offerte par les reproches du Tasse, témoignages de l'existence, encore quelques décennies plus tôt, d'une liberté d'expression restée ensuite sans égale pendant plus de quatre cents ans, que j'avais entrepris, il y a une douraine d'années, une analyse de l'érotisme dans le Roland furieux dont je devrai ici me contenter de rappeler succinctement les grandes lignes, avant, pour finir, de m'arrêter plus longuement sur un ou deux passages particulièrement représentatifs de l'art de conter de l'Arioste.

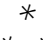

Avant toute chose, il convient de préciser que, même si l'amour et ses problèmes y sont omniprésents, on ne trouve dans le Roland furieux ni une exploitation systématique - ou seulement insistante - de sujets ou de situations relevant du sensuel, du sexuel, du voluptueux et de la jouissance, ni ce "goût marqué, excessif ou pathologique pour les choses sexuelles» qu'évoque le Dictionnaire alphabétique et analogique de la langue française de Paul Robert à la rubrique "Erotisme». Il est sans aucun doute plus juste, à propos du poème de l'Arioste, de parler d'une érotique, dans un sens proche de celui que donne à ce terme René Nelli, lorsqu'il définit "l'Erotique provençale (ou Amour provençal) [comme] l'ensemble des théories et des conduites socialisées qui, dans le Midi de la France et dans les divers pays d'Europe influencés par la culture occitane (Catalogne, Italie, France du Nord, etc.), ont pendant trois cents ans - du début du XII e siècle à la fin du $X I V^{e}$ - régularisé la tendance sexuelle et donné un sens nouveau à l'idée d'amour ». ${ }^{13}$ Ce n'est en effet pas un hasard si, au chant I, strophe 43, de la version finale de son poème, l'Arioste insère dans la tirade bien peu dans son tempérament que le païen Sacripant se récite à lui-même dans le faux espoir d'oublier une blessure d'amour qui est surtout blessure d'amour-propre d'un mâle supplanté, une précision inexistante dans l'édition de Is I6 sur le prix de la virginité, dont la jeune fille doit avoir plus grand soin que de ses beaux yeux et de sa vie même: formulation heureuse d'un idéal et d'une conception de l'amour et des rapports entre amants bien perceptibles dès la première version du poème, non seulement dans le comportement de la belle princesse du Cathay, comme dans celui d'Isabelle - "parangon de continence» (XXIX,20) crée de toutes pièces par l'A rioste, en hommage peut-être à Isabelle d'Este -, ou encore de Bradamante, la "vierge sage» (XXII,34), future génitrice de la lignée princière de Ferrare, mais aussi dans la conduite exem- 
plaire de Roland, le héros éponyme, amant irréprochablement maître de ses instincts, voué à la protection respectueuse de la "fleur [qu'il] voulai[t se] garder intacte 》 (VIII,77) jusqu'à l'beure du consentement mutuel et du mariage. Et c'est, semble-t-il, autour de cette érotique idéale - d'ascendance chevaleresque, mais teintée sans doute tant de moralisme chrétien que d'un "féminisme» (muliebrismo) hérité des divers courants poétiques qui, du Dolce Stil Novo au pétrarquisme et au néo-platonisme, ont marqué la culture italienne des siècles précédents - que s'édifie dans le Roland furieux une casuistique amoureuse grossièrement subdivisible en deux types opposés de comportement: le comportement relevant d'un effet pervers du code érotique orthodoxe, conduisant Roland à une folie à la (dé)mesure du personnage, qui n'est pas simplement l'issue extrême d'une passion non payée de retour, mais l'aboutissement tragique d'un respect aussi total que radicalement déçu de conventions sociales inhibitrices, l'explosion violemment libératrice d'une sensualité trop longtemps et inutilement refoulée; et, aux antipodes de cette byper-fidélité à un code que beaucoup considèrent comme suranné ou ignorent, une série beaucoup plus fournie et variée de comportements plus ou moins consciemment transgressifs, naturellement typiques des grands héros païens, brutaux et insensibles par définition aux raffinements courtois, mais qui ne sont - loin de là - l'apanage ni des païens ni des seuls hommes.

C'est logiquement dans l'évocation du comportement des personnages de cette seconde catégorie que l'on peut s'attendre à voir le langage du poème glisser le plus aisément vers l'érotisme, encore que l'on puisse en trouver aussi ailleurs et qu'en revanche toute situation luxurieuse ne donne pas nécessairement lieu, dans le Roland furieux, à une description érotique. L'Arioste n'est pas l'Arétin; et telles scènes que l'auteur des Ragionamenti n'aurait pas manqué de décrire avec complaisance, peuvent, dans le Roland furieux, se réduire à une simple, fugitive évocation. Ainsi Dalinde résume-t-elle pudiquement dans l'expression de jeu amoureux (" amoroso gioco 》: V,II) ses nombreuses rencontres nocturnes avec Polynesse. Et la nuit que Doralice, enlevée par Mandricard, accepte sans trop se faire prier de passer avec son ravisseur, se traduit, sous la plume du poète, par une strophe qui est un chef-d'cuvre d'élusion et d'allusions discrètes:

Ce qu'ensuite il advint, quand la nuit fut venue, entre elle, Doralice, et le fils d'Agrican, je ne puis vous le dire en toute certitude; si bien qu'il appartient à chacun d'en juger : qu'ils s'accordèrent bien on a tout lieu de croire, car quand le lendemain joyeux ils se levèrent, Doralice bien fort remercia le berger de l'avoir honorée d'un lit dans sa demeure. (XIV,63) 
ÉROTISME DISCRET DE L'ARIOSTE

L'Arioste, toutefois, est loin d'être toujours à ce point allusif; et lorsqu'il évoque plus concrètement des étreintes amoureuses ou l'acte sexuel proprement dit, au stade tant du désir que de l'accomplissement, son mode d'expression, comme chez. l'Arétin, Berni et beaucoup d'autres avant eux, est bien évidemment la métaphore. Ainsi, la double équivalence, d'abord entre la rose et la vierge, puis entre la cueillette de la rose et la défloration de la vierge, affichée dès le premier chant dans le lamento de Sacripant (I,42-43), inaugure tout un réseau métaphorique minimal, à la fois rudimentaire et sans originalité, mais doté de la transparence et de l'efficacité expressive des vieux clichés connus de tous: où la rose, une fois devenue métaphore, non plus de la vierge, mais du siège physiologique de sa virginité, peut alterner avec des synonymes métaphoriques tels que la fleur ou encore le fruit à cueillir; quitte à rétablir ailleurs une distinction entre ces deux derniers termes, comme dans la scène des retrouvailles de Roger et Bradamante (XXII,32-34), où les " premières fleurs» que Roger cueille sur les lèvres de la belle ne sont rien de plus que des baisers, sans commune mesure avec les «derniers fruits» que Bradamante, dans son irréprochable sagesse, déclare ne vouloir accorder qu'après le mariage. Tout compte fait, donc, les métaphores végétales ne sont guère que des lieux communs, euphémismes pudiques relevant d'un érotisme pour ainsi dire de bonne compagnie.

Plus hardies, déjà, sont quelques images éparses empruntées au registre des activités humaines, comme par exemple les métaphores militaires (XXV,6869; XXXV,75-77; et surtout XIX, passim): inévitables dans un poème dont la guerre constitue l'épine dorsale, même si elles y sont moins fréquentes que dans les comédies de l'Arioste, héritées de la tradition latine. ${ }^{14}$ Mais c'est surtout au registre animal que font appel les scènes du Roland furieux les plus proprement qualifiables d'érotiques. Ainsi le récit de l'assaut que livre en vain le vieil ermite libidineux à Angélique, préalablement endormie par ses soins, repose-t-il entièrement sur l'image du destrier, métaphore évidente du phallus (VIII,49-50). La même métaphore équestre reparaît à la fin du chant $X$, lors du second assaut libidineux infligé à l'béroïne - par Roger, cette fois-ci -, et à nouveau dans le commentaire de la première strophe du chant suivant, où une comparaison associe le "fougueux destrier " à l'ours friand de miel que rien ne saurait retenir. Elle reviendra ensuite en plusieurs points du poème, avec des sens variables liés au symbolisme complexe du cheval, largement attesté dans d'autres poésies équivoquées contemporaines. ${ }^{\text {is }}$

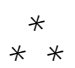

Si utile qu'il puisse être pour la comprébension des artifices utilisés pour camoufler sous des propos formellement honnêtes les scènes et les situations les plus scabreuses, un relevé d'expressions coupées de leur contexte ne saurait 
cependant suffire à rendre compte de l'usage que fait l'Arioste d'un érotisme dont elles ne constituent le plus souvent que le temps fort: l'acmé, à la fois censuré et souligné par la ou les figures employées, de récits eux-mêmes foncièrement transgressifs de la bienséance, mais jusque-là racontables et racontés en un langage ordinaire.

Cela est particulièrement évident dans les dix ou donz̨e récits dans le récit plus ou moins nécessaires au déroulement de l'intrigue, généralement connus sous le nom - en partie abusif - de "nouvelles du Roland furieux », et où, après d'autres - Luigi Pulci, Matteo Maria Boiardo, le Cieco da Ferrara -, l'Arioste sait pouvoir bénéficier d'une désormais traditionnelle marge de liberté, sinon de licence. ${ }^{16}$ Et ce n'est pas un hasard si, parmi ces récits conçus comme des sortes de pauses plaisantes, boccacesques, dans la relative monotonie des prouesses chevaleresques, les plus hardis sont également les plus marginaux: de l'épisode de Richardet et Fleur-d'épine, raconté à Roger sous le prétexte faiblement fonctionnel d'illustrer la ressemblance entre Bradamante et son frère jumeau et les quiproquos qu'elle entraîne (XXV,22-70); à l'odyssée amoureuse du roi Astolphe et de Jocond, plus connus sous l'appellation de "nouvelle de Flammette », du nom de la jeune amante à laquelle les deux béros doivent leur expérience décisive de la nature féminine, précautionneusement autant qu'ironiquement présentée par l'auteur lui-même comme une excroissance inutile à la trame de son poème (XXVIII); à la double bistoire, d'Argie et du juge Anselme (XLIII,72-I43), expressément racontée à Renaud comme un doublet de l'bistoire des mésaventures conjugales du Signore de Mantoue qu'il avait entendue un peu plus tôt de la bouche même de la victime, artisan de son propre malheur (XLIII,II-46).

La lecture suivie de ces trois épisodes, dont le dernier ne comporte pratiquement plus la moindre métaphore obscène, montre bien que la paillardise, chez. l'Arioste, n'est pas une fin en soi et qu'il ne se précipite pas systématiquement, comme plus tard l'Arétin des Ragionamenti, sur la moindre occasion de peinture licencieuse. L'utilisation des métaphores ou autres images de substitution répond dans le Roland furieux à des critères narratifs plus raffinés: soit que leur apparition coüncide avec ce que Calvino appelait le «moment sexuellement culminant» d'un récit où l'auteur a soigneusement ménagé ses effets, préparé et alléché un public partagé entre le désir empreint de voyeurisme de jouir de la scène et celui de voir jusqu'où le poète osera pousser la liberté; soit que ces images fleurissent en des points bien déterminés, avec une fonction précisément calculée, à la fois narrative et, pour ainsi dire, déontologique: l'emphatisation rhétorique et la scansion des temps forts d'un récit érotique diffus, en même temps que le camouflage, sous un langage transparent, des passages où l'interdit moral ne peut être bravé impunément en langage clair. ${ }^{17}$ 
Si, dans ces sortes d'intermèdes semi-autonomes que sont les "nouvelles", où tend à se concentrer la matière la plus lascive, les écarts de langage sont ainsi toujours judicieusement contrôlés, à plus forte raison le sont-ils dans le reste $d u$ poème, où, jusque dans l'évocation du royaume de la fée Alcine, pourtant incarnation expresse de la luxure, l'Arioste ne se départit pas d'une réserve de bon aloi. L'érotisme est en effet des plus discrets tant dans la présentation du "paradis » peuplé de jeunes gens et de petits amours où pénètre Roger (VI,72 et suiv.), que - comme on le verra dans un instant - dans la description des charmes d'Alcine. Il croît insensiblement ensuite, du repas où les amants se chuchotent à l'oreille leurs secrets, au coucher du héros

dans le lin parfumé,

qui paraissait sorti de la main d'Arachné $(\mathrm{VII}, 23)^{18}$

et son attente anxieuse de la venue de la fée, qui longuement se parfume et s'apprête pour la nuit. Puis le style devient plus suggestif dans l'évocation des vêtements très succincts que porte Alcine à son arrivée, du léger manteau qu'elle abandonne aussitôt, ne gardant plus sur elle qu'une chemise des plus transparentes:

voile clair et fin

qui, derrière et devant, ne la couvrait pas plus

qu'un verre transparent les roses et les lys. (VII, 28)

L'érotisme culmine ensuite, naturellement, dans l'étreinte des deux amants enlacés comme lierre à l'arbre, cueillant sur les lèvres l'un de l'autre des fleurs plus suaves que n'en produisent les Indes ou l'A rabie, et brusquement s'épuise dans une pirouette aussi malicieuse que triviale, par laquelle l'A rioste frustre l'auditoire qu'il a ainsi graduellement appâté, lui signifiant son refus de narrer la suite des ébats:

Du plaisir qu'ils prenaient, c'est à eux de parler,

car ils avaient souvent deux langues dans leur bouche. (VII,29)

Plus encore que dans les scènes proprement amoureuses, ce parti pris qu'adopte l'Arioste - à plus forte raison lorsque sont en cause les principaux béros du poème - de décevoir ainsi son public après l'avoir consciencieusement échauffé et tenu en haleine, est patent dans les portraits et dans les très rares nus du Roland furieux. Et - si on veut bien me pardonner de revenir en arrière, une vingtaine de strophes avant celle que je viens de citer - exemplaire à cet 


\section{Paul Larivaille}

égard est la minutieuse, plus que toute autre analytique, description d'Alcine au moment où Roger la découvre, souverainement belle parmi les beautés de sa cour, «comme le soleil est plus beau que toute étoile» (VII,Io).

$$
\text { * } *
$$

D'accord pour identifier dans ce portrait d'Alcine (VII,II et suiv.) tout un arsenal de réminiscences littéraires et de clichés conventionnels, la critique s'est divisée au niveau du jugement, les uns en condamnant l'intellectualisme et la froideur tandis que d'autres y exaltaient une contemplation détachée, exempte de toute sensualité, une sorte de "jouissance olympienne de la forme 》. ${ }^{19}$ Les uns et les autres, toutefois, prisonniers de leur subjectivité et de leur culture d'bommes du XIX ${ }^{e}$ ou du XX siècle, semblent n'avoir pas pris en compte dans leurs appréciations le rapport entre l'auteur et le public pour lequel il écrivait: le parti, notamment, que le poète pouvait tirer - sciemment sans doute - de la suggestivité des lieux communs, d'autant plus suggestifs qu'ils ne faisaient en l'occurrence que réveiller et flatter une image stéréotypée de la beauté enracinée dans la culture de toute une époque. L'important, cela étant, est donc moins de dénoncer ou interpréter anachroniquement le recours à des conventions auxquelles l'A rioste ne pouvait et probablement ne voulait pas se soustraire, que d'observer comment il en joue et éventuellement se joue d'elles en même temps que du public auquel il les ressert.

Dès la référence initiale à la peinture, le portrait affecte les allures d'un traité d'esthétique, attentif à la définition des canons de la beauté parfaite plus qu'à l'évocation subjective de ses effets. ${ }^{20}$ La tête est détaillée en trois strophes: une chevelure blonde tombant sur de joues "d'une couleur mêlant les troènes aux roses »; puis un front d'ivoire de justes proportions; deux sourcils noirs finement arqués surplombant deux yeux noirs eux aussi; un nez bien centré et sans défaut; une bouche de cinabre entre deux légères commissures semblables à de petites vallées, laissant voir deux rangées de "perles fines》. L'image résultante peut à juste titre apparaître excessivement conventionnelle et froide, surtout à des siècles de distance, aux yeux de lecteurs avertis de ses antécédents littéraires et portés à n'y voir qu'un amalgame de réminiscences sans originalité. Mais elle ne l'était sans doute pas pour des hommes de la Renaissance, capables, à partir d'une énumération forcément allusive, de reconstituer concrètement les éléments d'une beauté bien présente à leur esprit; d'autant que leur imagination ne pouvait qu'être excitée par un crescendo de notations plus proprement affectives, perçues aujourd'bui, avec le recul, comme d'abstraits sinon insipides poncifs, mais qui pour le public du temps étaient probablement des stimuli adaptés à sa sensibilité et à une culture où ce qui n'est plus pour nous que plates réminiscences correspondait encore à des idéaux bien vivants: 
Érotisme DisCret DE L'Arioste

de la banale superlativisation du blond de la chevelure, plus que laquelle « aucun orne pourrait resplendir et briller ", à laparadoxale et banale à la fois comparaison des yeux noirs à «deux clairs soleils», à la suggestive mais sans doute pas nouvelle constatation que laperfection du nezest telle que "l'envie n'y voit rien qui soit à corriger ", et pour finir au quatre vers qui prolongent la description de la bouche, dans l'évocation peut-être sans originalité mais délicieusement désuète des paroles aptes à vaincre les cours les plus rustres qui en émanent, et du sourire si suave "qu'il déclôt à son gré le paradis sur terre ».

Bref, même si les ingrédients en sont on ne peut plus traditionnels, l'effet que devait produire sur un auditoire contemporain le portrait d'Alcine était, lui, on ne peut plus concret. Ce à quoi, de lieu commun en lieu commun, de cliché en cliché, procède l'Arioste, c'est à une graduelle mise en condition de son public: il le prépare, par un reste d'indispensable opportunisme courtisan, à excuser comme inévitable la chute de Roger, mais surtout, par delà les excuses, à goûter sensuellement les étapes suivantes - évoquées plus haut - de l'inexorable séduction du héros par la magicienne. Aussi, du visage, amorce-t-il une lente descente vers des parties plus secrètes de la personne d'Alcine. Du cou et de la gorge il commence par souligner la parfaite blancheur (" son beau cou est de neige et sa gorge de lait »). Un second vers, passant du teint à la forme, note le galbe du cou et la plénitude épanouie de la poitrine. Les yeux s'arrêtent ensuite avec plus d'insistance (trois vers) sur les seins «faits d'ivoire eux aussi ", qui vont et viennent dans un mouvement déjà subtilement érotique comme, sur le rivage, houle soulevée par la brise. Puis, brusquement, renonçant à son "omnivoyance», l'auteur laisse le public ainsi alléché sur sa faim d'en savoir (voir!) plus, concluant avec une malice calculée qu'Argus lui-même ne pourrait en apercevoir davantage, mais que l'

on peut aisément juger que correspond

à ce qui apparaît tout ce qui est caché. (VII,I 4)

Et la déception de qui écoute ou lit est d'autant mieux orchestrée que l'A rioste consacre une demi-douzaine de vers supplémentaires à insister sur l'excitante perfection des extrémités dépassant des vêtements: les bras justement proportionnés, la main délicatement effilée; et surtout « un petit pied nerveux et des plus rondelets» (VII,IS), dont le potelé suggestif est le fruit d'une trouvaille tardive du poète. ${ }^{21}$

Ce portrait en pied d'Alcine est en même temps l'archétype de la beauté telle que la conçoit l'A rioste et le premier exemple de cet érotisme contenu dont il ne se départira pas dans ses évocations successives de la perfection féminine.

Les éléments constitutifs de cette beauté effectivement ne varient guère au long du poème. Angélique, dont les charmes ne sont que très rarement détaillés, est 
comme Alcine une blonde aux yeux noirs, et si passagèrement - résultat sans doute de sa fuite éperdue - on ne perçoit de ses joues que le vermeil (XII,33), les couleurs de son visage au repos et de son corps sont identiques à celles de la magicienne: ses mains contemplées par Roger $(X, 98)$ sont « d'ivoire bien poli》 et son visage vu en songe par Roland (VIII,80) est lui aussi d'un "bel ivoire",, tout comme le front d'Alcine (VII,II); sur ses membres alternent la blancheur du lys, équivalent chromatique du troène, et le vermeil des roses $(X, 95)$; et on voit qu'une larme, glissant

sur les troènes blancs et sur les fraîches roses, baignait de sa rosée les fermes petits seins et que le vent faisait voler ses cheveux d'or. $(\mathrm{X}, 96)$

De la même manière, sur le visage d'Isabelle sanglotante, les larmes coulaient entre des lys et des roses (XII,g4); et, dans la troisième édition du poème encore, Olympe, la dernière venue des grandes bérö̈nes de l'Arioste verse un "ruisseau 》 de larmes «coulant parmi les fleurs et blanches et vermeilles》 (XI,66).

Que l'Arioste fasse en ce domaine si peu d'efforts pour se renouveler prouve à l'évidence que l'on se trouve en présence d'un authentique stéréotype, d'une représentation de la femme ancrée dans l'imaginaire collectif, qui non seulement impose des limites assez strictes à l'originalité du poète, mais probablement fait dépendre l'efficacité et la suggestivité de l'écriture poétique du respect de canons esthétiques et expressifs fonctionnant comme un code difficilement contournable.

A ces exigences de l'esthétique ambiante, le portrait d'Alcine montre que s'ajoutent, pour l'Arioste, celles de la morale, et plus précisément d'une certaine décence. Il se joue de l'attente du public et de la censure éventuelle en suggérant à demi-mot les attraits des parties de la magicienne qu'il ne peut pas ou ne veut pas découvrir, comme il ironise ensuite, en présence d'une Angélique nue qu'il se garde de décrire autrement que par quelques conventionnelles généralités, sur la pudeur comique de la princesse qui

aurait de ses mains abrité son visage, mais celles-ci étaient liées au dur rocher; $(\mathrm{X}, 99)$

comme il badinera encore plus tard sur Olympe, qui

en cachant de son mieux sa poitrine et son ventre,

$[\ldots]$ livre encor plus ses hanches et ses reins, 
ÉrotisMe DisCRET DE L'Arioste

en même temps que sur la hâte du très pudique Roland, de rentrer au port pour couvrir au plus tôt la belle de quelque vêtement $(X I, 59)$.

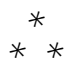

Pourtant, d'Angélique à Olympe, une indéniable évolution s'est produite, d'autant plus remarquable que, dans la troisième édition du Roland furieux, l'A rioste a sciemment inséré la première et la seconde libération de la fille du roi de Hollande par Roland de part et d'autre de la libération d'Angélique par Roger: dans le but évident d'enrichir son poème d'un de ces parallélismes qu'il affectionne, faits d'analogies mais aussi de différences que le lecteur est ainsi invité (pour ne pas dire provoqué) à détailler. Une quinzaine d'années après la diffusion de la première version, l'Arioste a visiblement acquis de la hardiesse. De la nudité suggestivement mais soigneusement voilée d'Alcine, et de celle sans voiles mais tout juste discrètement suggérée d'Angélique, il en vient sur le tard au seul véritable nu que contienne son poème (XI,67 et suiv .).

Après l'évocation babituelle du visage éploré et de l'amour qu'en éprouve aussitôt le roi d'Irlande, le poète, sans même se masquer derrière le regard détaché de Roland, entreprend de décrire Olympe nue avec une témérité et un empressement inusités. Postulant d'emblée qu'Olympe « avait de ces beautés / que l'on voit rarement", il recourt tout aussitôt à une sorte de prétérition déguisée pour se débarrasser sommairement, en moins de quatre vers, des lieux communs obligés de tout portrait et manifester hardiment son intention de fixer plus longuement son regard sur les autres parties $d u$ corps:

car son front était beau,

mais encore ses yeux, ses joues et ses cheveux,

sa bouche et puis son nez, sa gorge et ses épaules;

et, si l'on descendait plus bas que les tétins,

les parties que souvent une robe recouvre

furent d'une excellence assez grande peut-être

pour qu'on les mit avant toutes celles du monde. (XI,67)

Avec la descente annoncée du regard commence, à la strophe suivante, une érotisation des plus gaillardes qui déborde vite de la simple visualisation de la scène. Un premier coup d'cil d'ensemble sur les "parties》 babituellement cachées de la belle glisse, en effet, en deux vers, de la constatation superlative qu'elles l'emportaient «en blancheur sur les neiges intactes», à l'affirmation qu'elles étaient " au toucher plus lisses (molli) que l'ivoire»; où l'allusion soudaine au toucher introduit une note étonnamment concrète: comme si, 


\section{Paul Larivaille}

entraîné par son imagination au comble du voyeurisme, le poète oubliait sa position de spectateur imaginaire et se prenait à livrer, à son corps défendant, des souvenirs d'une beauté palpable et personnellement palpée. La charge érotique produite par cette évocation tactile trouve confirmation immédiatement après, dans les deux premiers vers consacrés aux seins, où l'image triviale du lait caillé que l'on vient de retirer des joncs où il était suggère elle aussi un contact de la main avec ces "petits seins ronds》 dont la forme (ritondette = littéralement "rondelettes») rappelle celle, d'une égale sensualité, du pied lui aussi « rondelet » d'Alcine (VII,IS). Puis la descente du regard entre les seins fait appel à l'image à la fois artificieuse et très concrète de "l'ombreuse vallée » entre "deux tout petits coteaux», que l'hiver aurait privée de ses ombrages et recouverte de neige; une utilisation érotique de la nature d'autant plus surprenante que de telles images renvoient habituellement, dans la littérature de l'équivoque, à des parties plus basses du corps: l'entrecuisse, et plus précisément parfois la vulve ou, comme suggère ici la forme hémisphérique des collines, le «sillon interfessier $》 .{ }^{22}$ Le recours à l'expédient de l'biver et de la neige, sans lequel l'image de la vallée ombreuse risquerait d'évoquer un espace entre les seins grotesquement sombre, sinon velu, traduit, dans son artifice, peut-être un jeu destiné à préparer sournoisement le public à la chute de la strophe suivante, en même temps qu'il renforce l'insistance sur la blancheur, couleur par excellence de la beauté pour les contemporains de l'Arioste, dont le retour insistant est probablement, cela étant, un facteur non négligeable de l'érotisation du portrait d'Olympe.

La strophe qui suit $(X I, 69)$, outre qu'elle abaisse le regard vers des parties jusqu'alors jamais explorées dans le Roland furieux (flancs, hanches, ventre et cuisses), associe à nouveau aux canons formels (le galbe des flancs et la platitude du ventre) une limpidité de miroir, qui confere à la peau du ventre une luminosité nivéenne des plus suggestives, et la franche blancheur des cuisses. Mais graduellement, à mesure que les yeux approchent de zones plus intimes du corps, se met en place un dispositif de freinage, puis de blocage, de l'élan érotique. Après l'évocation des flancs, des hanches et du ventre, l'auteur introduit un verbe ("paraissaient faits»), comme s'il en avait terminé avec l'énumération des sujets et entendait poursuivre sa phrase. Puis, comme s'il se ravisait soudain, il ajoute après le verbe un quatrième sujet ("ses cuisses blanches»), qui se trouve ainsi à la fois mis en évidence par la rupture de construction et isolé comme une chose quasiment indicible, ajoutée du bout des lèvres, après une bésitation, comme une sorte d'ultime limite infranchissable. Le vers biparti qui suit (dans le texte italien), reprenant d'abord la proposition précédemment laissée en plan pour préciser que toutes les parties énumérées paraissaient faites au tour par Phydias, puis surenchérissant dans le second hémistiche qu'elles auraient pu être l'cuvre d'une main encore "plus experte », 
Érotisme DisCRET DE L'Arioste

dans le même temps et plus encore qu'à une superlativisation des attraits d'Olympe, semble viser à un prolongement graduel de la suspension de l'énumération précédente; jusqu'à la transformer en une rupture habilement renforcée par la question que l'auteur, feignant l'bésitation, pose aussitôt après au public:

De ces parties du corps dois-je aussi vous parler que cependant en vain elle voulait cacher?

Dès lors que l'Arioste a ainsi lanterné son auditoire, avec un luxe de formules dilatoires proportionnel à la témérité de ce qu'il avait semblé promettre et à l'attente accrue qu'il avait suscitée, tout est prêt pour une pirouette finale analogue à celle par laquelle il avait une vingtaine d'années plus tôt mis brutalement un terme à la description des charmes d'Alcine (VII,I4):

En somme je dirai que de la tête aux pieds

ce que beauté peut être en elle se voit toute. (XI,69)

Les deux strophes suivantes achèvent de consacrer le renoncement au concret, concluant la célébration d'Olympe par deux comparaisons avantageuses pour elle avec des beautés célèbres de l'Antiquité: celle d'Hélène, que Pâris, aux dires de l'Arioste, n'aurait pas enlevée s'il y avait eu à Troie quelque femme comparable à la fille du roi de Hollande; et celle des cinq belles Crotoniates prises pour modèles par Zeuxis, qu'Olympe aurait à elle seule pu suppléer, "car toutes les beautés en elle se trouvaient» (XI,7I). Cette chute par le biais d'un transfert dans le champ non compromettant des réminiscences d'une antiquité semi-légendaire differe certes de celle du portrait d'Alcine, où le poète avait trouvé dans les extrémités des membres dépassant des vêtements (les bras, les mains et les pieds, absents du portrait d'Olympe) un moyen élégant de suggérer la perfection des parties cachées du corps et d'entretenir ainsi babilement la tension sensuelle provoquée dans le public. Mais par delà ces différences liées contextuellement à l'écart entre les situations, les personnalités et les rôles assignés aux deux hérö̈nes, on ne peut que constater l'invariabilité d'un procédé qui est en quelque sorte emblématique du comportement de l'Arioste vis-à-vis de la matière érotique : à deux décennies de distance, à une érotisation savamment graduée qui émoustille progressivement la sensualité du public, créant en lui une attente de détails plus scabreux et savoureux, succède, au moment crucial, une même déception ironique de l'attente. 
Seule reste remarquable, de la première à la troisième édition du Roland furieux, la hardiesse accrue dans le dévoilement du corps, à mettre en rapport avec une évolution générale de l'auvre qui ne se limite pas aux seuls sujets touchant à l'amour et à la sexualité, mais, au fil des passages et épisodes ajoutés par l'auteur, se caractérise par un baussement relatif mais général du ton, par une tendance à une peinture à la fois plus «engagée » et plus crue des choses, à laquelle n'échappent ni les nouveaux personnages tardivement introduits, ni leurs comportements, ni leurs aventures. Dans un tel contexte, le nu d'Olympe relève sans doute moins d'un glissement général de la société de l'époque vers une plus grande permissivité, que d'une évolution personnelle de l'Arioste: d'une liberté d'expression qui s'est considérablement aiguisée au fil des années, avec l'assurance croissante que lui donnait en premier lieu le succès de son poème, mais aussi son expérience d'bomme et d'écrivain, de serviteur apprécié et respecté des princes de Ferrare en même temps que d'auteur de Satires et de comédies plus ouvertement mordantes que ne l'avaient été les premières; sans oublier l'âge, dont les atteintes exacerbaient peut-être sa libido, émoussant quelque peu sa retenue débonnaire et sa prudence d'autrefois. L'écart, toutefois, entre le portrait d'Alcine et le nu d'Olympe n'en reste pas moins très contrôlé. L'Arioste vieillissant ne se départit pas de cette malicieuse discrétion qui, après comme avant les ajouts de I532, demeure le caractère dominant de l'érotisme dans le Roland furieux : un érotisme qui, loin des jeux systématiques de cache-cache ou de devinettes entre initiés couramment pratiqués dans la poésie équivoquée de l'époque, n'a visiblement pas d'autre but que de corser le récit chevaleresque de gaillardises à la fois transparentes et soigneusement mesurées. Et la déception de l'attente de l'auditoire et des lecteurs à laquelle il vise de façon pratiquement invariable, n'est au fond qu'un bel exemple de plus de cette fameuse ironie qui régit de bout en bout les rapports de l'Arioste non seulement avec la matière de son poème, mais avec son public. 
ÉrotisMe Discret de L'Arioste

I. Ce titre, qui veut être une honnête indication de source, renvoie à dessein à celui d'une recherche beaucoup plus vaste et systématique, Les Jeux de l'amour dans le "Roland furieux", ou l'érotisme discret de l'Arioste, parue il y a une dizaine d'années dans le vol. il des publications du Centre Interuniversitaire de Recherches sur la Renaissance Italienne, $A u$ Pays d'Eros. Littérature et érotisme en Italie de la Renaissance à l'âge baroque ( ${ }^{\mathrm{e}}$ série), Paris, Université de la Sorbonne Nouvelle, i 988, p. 53-I 44. La seconde partie de mon exposé sera en effet un rappel des grandes lignes de ce long travail de recherche dont - avec un certain nombre de retouches dont je rendrai compte plus loin - j'extrairai, dans la partie finale, deux exemples particulièrement caractéristiques à mes yeux de l'art de l'Arioste.

2. J. Toscan, Le Carnaval du langage. Le lexique érotique des poètes de l'équivoque de Burchiello à Marino (XV'e-XVII siècles), Lille, Presses Universitaires de Lille, I 98 I, 4 tomes, 2 I 5 I pages. Sur Burchiello et Laurent, ch. II et III, p. 59-I 3 I.

3. Lettre du 8 mars I I I9, d'Alfonso Paolucci, ambassadeur de Ferrare à Rome, au duc Alphonse I d'Este (in Commedie del Cinquecento, a cura di A. Borlenghi, Milano, Rizzoli, I959, I, p. 975) : «'l papa ne rise assai gagliardamente con gli astanti » (selon Paolucci, seuls certains Français se «scandalisèrent quelque peu»!). La comédie fut traduite en français par Jean Pierre de Mesmes, sous le titre La Comédie des Supposez (Paris, Estienne Groulleau libraire, I5 52). Le titre et la première partie du prologue jouaient sur les différents sens du verbe supporre accessibles, à l'époque, à des latinistes avertis : «substituer, échanger, mettre à la place de», mais aussi (cf. le sens premier du latin supponere, «mettre dessous »... et suppositorium, «suppositoire»!) «sodomiser ». Plus tard, dans le prologue de la même comédie réécrite en vers, l'Arioste multipliera les jeux de mots sur le même thème, feignant de démentir l'interprétation licencieuse qui fait rire les spectateurs: «Mais vous riez? Oh, qu'ai-je bien pu vous dire de risible? Ah oui, j'imagine d'où vous doit venir ce rire. Vous croyez que je veux vous dire quelque cochonnerie, ou vous la donner en spectacle» (vers I I-I s). Un peu plus loin, après s'être défendu (comme dans le premier prologue) de vouloir rivaliser avec les livres illustrés de postures de la femme-écrivain grecque Eléphantis, dont Suetone (Vie de Tibère, XLIII) raconte que l'empereur conservait un exemplaire dans sa retraite de Capri, l'Arioste ajoutera que ses propres « suppositions » n'ont rien à voir avec celles que l'on a vu reparaittre «de nos jours dans la Sainte Rome, et [que l'on a] faites imprimer sur des feuilles plus belles qu'honnêtes, afin que le monde entier en puisse profiter» (vers 3 I-34): allusion probable à la publication, en I 524 ou I 525 , de dessins érotiques de Jules Romain gravés par Marc-Antoine Raimondi, au bas desquels l'Arétin avait ensuite écrit ses Sonnets luxurieux (cf. L'Aretin, Sonnets luxurieux sur les XVI postures, trad. de l'italien par A. Bonneau et P. Larivaille, préface et notes de P. Larivaille, Paris, Payot et Rivages, 1996).

4. Voir la liste des «bernesques» italiens donnée par J. Toscan, Le Carnaval du langage, cit., I, p. I38-39; et ibid., IV, p. 2070-2082, passim, outre la bibliographie des œuvres des plus importants d'entre eux, les ouvrages collectifs (p. 2077) où ont été publiées les poésies burlesques des autres. En ce qui concerne la France, Toscan renvoie à : P. Toldo, Etudes sur la poésie burlesque française de la Renaissance, in Zeitscbrift für Romanische Pbilologie, I90I ; et F. BAR, Le Genre burlesque en France an XVII siècle, Paris, D’Artrey, ig6o.

5. C. Du Marsais, Traité des tropes, ou des différens sens dans lesquels on peut prendre un même mot dans une même langue (1730), II, xv, L'euphémisme (les italiques dans le texte sont de moi). Je cite d'après «Le Nouveau Commerce», cahiers i 5 -16, printemps-été I970, p. I45. Voir J. Toscan, Op. cit., t. I, p. I43-44, et plus largement tout le par. II de l' «Introduction à la deuxième section », p. I4I-46.

6. J. Toscan, Op. cit., p. I638 (où est rappelée une bévue de Vittorio Cian signalée précédemment, p. I615-16). Ailleurs sont corrigées des erreurs de Virgili et Sorrentino, auteurs des deux ouvrages les plus connus sur Berni; ou du grand philologue Michele 


\section{Paul Larivaille}

Barbi (p. 664-65); ou de Moncallero, biographe du cardinal Bibbiena (p. 2054, n.10), etc.

7. Voir ibid., I, p. I44, ce que Toscan dit de l'euphémisme simulé, « une habileté littéraire qui permet souvent de donner plus de sel et plus de relief à des gaillardises qui, sans lui, tomberaient à plat », grâce précisément à la «transparence » des termes qui «permettent aux conteurs de facéties ou aux auteurs de comédies de suggérer des objets ou de peindre des situations dont le caractère scabreux est par lui-même comique, sans qu'il soit nécessaire de demander au public un grand effort de compréhension et tout en observant les règles, au moins formelles, de la décence».

8. Pour ne citer que les deux premières et la dernière des cinq comédies, voir en particulier, passim : Cassaria en prose (I 508), III, sc. 3 et 4 ; Suppositi en prose (I 509) : prologue ; I, sc. 4 ; II, sc. 2 ; III, sc. I et 2 ; IV, sc. 2 ; V, sc. 5 ; Lena (I 28 -29) : second prologue ; II, sc. 3 ; IV, sc. 9, ainsi que divers jeux de mots épars du valet Corbolo.

9. P. Larivaille, Pietro Aretino, Roma, Salerno Editrice, 1997, ch. V, par. 3-4, p. I9I et suiv. ; L'Aretin, Ragionamenti, I, Edition bilingue ; Introduction, traduction et notes de P. Larivaille, texte établi par G. Aquilecchia, Paris, Les Belles Lettres, 1998.

io. P. Larivaille, Pietro Aretino, cit., ch. VII, p. 296-307, passim.

I I. Voir A. Rochon, Les Pièges de l'érotisme dans la "Jérusalem délivrée », in (collectif) Au Pays d'Eros. Littérature et érotisme en Italie de la Renaissance à l'âge baroque ( ${ }^{\mathrm{e} r e}$ série), Paris, Centre Interuniversitaire de Recherche sur la Renaissance Italienne, Université de la Sorbonne Nouvelle, I986, p. 7I-171 ; et N. Jonard, L'érotisme dans la "Jérusalem délivrée», in «Studi Tassiani », no 32,1984, p. 43-62.

I 2. Discorsi dell'arte poetica..., dans Torquato TAsso, Scritti sull'arte poetica, a cura di E. MAZZALI, Torino, Einaudi, I977, t. I, p. 47-48, passim ; et Discorsi del poema eroico, ibid., t. II, p. 3 10-I I, passim.

I3. R. NelLi, L'Erotique des troubadours, Paris, Union Générale d'Editions (10/I 8), I974, vol. I, p. I I. Un peu plus loin (p. I 3), l'auteur précise ainsi sa définition liminaire : «L'Erotique provençale est sans doute le système le plus efficace qui ait été imaginé pour réduire l'instinct sexuel tout en l'exaltant, et pour permettre ainsi à l'amour de se dépasser selon sa propre loi, de se purifier en s'approfondissant».

I4. Sur la récurrence du langage figuré militaire - hérité de Térence en particulier - dans les comédies de l'Arioste, voir Ariosto, Commedie, a cura di A. Casella, G. Ronchi, E. Varasi, Milano, Mondadori, I974, p. Io I 5, n. 57 . Pour plus de détails sur le chant XIX du poème, où l'exploitation de cette veine métaphorique est la plus insistante, voir P. LariVAILLE, Les Jeux de l'amour dans le "Roland furieux», cit., p. 79-8 I.

i s. Cf. J. Toscan, Op. cit., Glossaire, IV, p. I677, où, à la rubrique Cavallo, sont enregistrés les sens de partenaire sexuel (giton ou femme) et de sexe (masculin le plus souvent, mais aussi féminin). Voir aussi ibid., p. I686, Destriero (métaphore du phallus), et plus particulièrement t. III, p. I 507-I 5 I I, l'analyse détaillée des différentes valeurs symboliques du cheval, qui est l'animal le plus fréquemment nommé dans les textes équivoqués.

I6. Les «nouvelles» avaient déjà été analysées en tant que telles par L. Di Francia, Novellistica, Milano, Vallardi, i925, II, p. 253-72. Voir également Ludovico Ariosto, Novelle del «Furioso», a cura di R. Battaglia, Milano, Universale economica, i950. Passibles de l'appellation de «nouvelles» sont les histoires de: Ginevra (V,5-74), Olimpia et Bireno (IX,22-56), Isabella (XIII,3-3I), Norandino et Lucina (XVII,25-68), la cité des femmes 


\section{ÉROTISME DISCRET DE L'ARIOSTE}

homicides (XIX,9-64), Gabrina (XXI,I I-66), Bradamante, Ricciardetto et Fiordispina (XXV,22-70), Astolfo, Giocondo et Fiammetta (XXVIII,4-74), la coutume en vigueur dans la «Rocca di Tristano» (XXXII,83-96), Lidia (XXXIV,II-43), Drusilla et Marganorre (XXXVII, 38-85), la « coupe enchantée» (XLIII, I I-46), Argie et Anselmo (XLIII,72-I 43). A noter que Di Francia n'évoque ni l'histoire de la cité des femmes homicides, ni celle de la coutume de la «Rocca di Tristano», qui ne sont peut-être pas à proprement parler des nouvelles, dans la mesure où elles ne sont pas construites autour d'une intrigue centrée sur un ou quelques héros bien définis. Mais sans doute serait-il illusoire de vouloir à toutes forces tracer une ligne de démarcation formelle trop nette entre des nouvelles caractérisées et des récits d'événements antérieurs considérés comme parties intégrantes de la trame du poème. D'autant que, parmi la douzaine de nouvelles retenues par Di Francia (selon des critères qu'il n'expose pas, mais qui prennent probablement avant tout, et légitimement, en compte l'unité de l'action et son «isolabilité» du contexte), il reconnait lui-même qu'une dizaine « sont savamment greffées sur les branches les plus robustes, tandis que deux autres sont racontées, sous une forme autonome, comme d'authentiques nouvelles 》 (p. 253$)$ : les deux seules nouvelles expressément présentées comme telles étant, on le sait, celle de Fiammetta et celle d'Argia et du juge Anselmo.

17. Pour une lecture plus approfondie des trois «nouvelles » évoquées, P. Larivaille, Les Jeux de l'amour..., cit., p. 90-I I 4 .

I8. Sauf indications contraires, les vers traduits en français que je citerai désormais seront empruntés, avec l'aimable autorisation du traducteur et de l'éditeur, à l'ouvrage suivant : L'Arioste, Roland furieux, Edition bilingue, Introduction, traduction et notes de A. Rochon, Paris, Les Belles Lettres, t. I (chants I-X), I 998, et t. II (chants XI-XX), en cours de publication. Ce recours à une traduction française nouvelle d'une très grande qualité explique les aménagements apportés au texte que j'avais publié il y a une dizaine d'années (voir supra, note I), dont sont extraites les dernières pages du présent exposé.

19. Voir par exemple le commentaire de Sapegno (Ludovico Ariosto, Orlando furioso, scelta e commento a cura di N. SAPEgno, Milano-Messina, Principato Editore, I969, $2^{\mathrm{e}}$ éd., p. 72-74), où se trouvent entre autres bien résumées les positions des uns et des autres.

20. Pour le détail, en particulier, des réminiscences de BocCace (Teseide, XII, 53 et suiv. description d'Emilia), Pulci (Morgante, XV,98 et suiv. : Antea) et Politien (Stanze, I,42 et suiv. : Simonetta) que l'Arioste amalgame dans le portrait d'Alcine, voir les notes de Cesare Segre dans son éd. du poème: Milano, Mondadori, I976, p. I 289, passim. Sur l'histoire des canons de la beauté féminine dans la culture et la littérature italiennes, du $\mathrm{XIV}^{\mathrm{e}}$ siècle au $\mathrm{xVI}^{\mathrm{e}}$, voir surtout l'important article de G. PozzI, Il ritratto della donna nella poesia d'inizio Cinquecento e la pittura di Giorgione, in «Lettere Italiane », a.XXXI, n. I, Gennaio-Marzo I979, p. 3-30.

2I. L'adjectif «ritondetto » n'apparait en effet que dans la $3^{\mathrm{e}}$ éd., à la suite d'un repentir bien venu de l'Arioste qui, dans la $2^{\mathrm{e}}$ éd., avait remplacé le «piccolino » (« tout petit») de la première par un peu exaltant «scarno » (« décharné », « maigre », au mieux : «sec »).

22. J. Toscan, Le Carnaval du langage, IV, Index : «Valle» et les passages de l'ouvrage auxquels renvoie la rubrique. 